\title{
The optimization of snowboarding motion and the design of a new snowboard course
}

\author{
F. Yang, H. Li \& A. Chen \\ Sichuan University, China
}

\begin{abstract}
According to the competition rules, the criterion of the score is the judgment of an athlete's action in the air as well as the height in the air. However, the action of an athlete and the height in the air is contradictory because one cannot reach the highest height and finish the maximum twist at the same time.

To find out the best method for athletes to get a high score, we designed a new shape of a snowboard course (half-pipe) in order to maximize the time of "flying" in the air as well as optimizing the twist of an athlete in the air by mathematical computation. What's more, through mathematical analysis, we designed the optimized time of an athlete soaring through the air from the wall of the half-pipe. The analysis of the motion and model of the snowboard course are also demonstrated by computer to make the conclusion more visual. Keywords: snowboarding, half-pipe, parabolic motion, differential equation.
\end{abstract}

\section{Introduction}

Snowboard movement is a stimulated event which has gradually gained in popularity in recent years. The scoring criteria of snowboard movement in the Ushaped pipe always consider two important facts-the height gain the in sky and the excellent action behave in the sky.

Actually, these two criteria require the athlete to have the ability to control himself and jump higher by taking advantage of the initial velocity and the jumping angle. On the other hand, for the audience, people wish the athlete to do great job for their entertainment.

So how to help the athlete gain the advantage is not only for athlete but also for the stadium builders. Now we will make a model to help them do better work and give them some advice. 


\section{Problem analysis}

For consideration of such a problem, we first must know well about the whole process of how the athlete moves up and does the action in the sky. And in other aspects, we all must get the criteria for the interesting movement like the score standard and some index about the half-pipe. So, after that we have already known something about the movement. It can be divided into three parts. The athletes move up by using the initial velocity. The snowboard gets attached to the pipe. So we must consider the friction. In this process, the kinetic energy turns to be as potential energy and heat caused by the friction.

The athletes move up to a certain point which is chosen by himself. Then he will jump up with such action that the back foot punch strong on the back side of the snowboard. The reaction force will give him another velocity in a certain direction. With the inertia and this velocity, the athlete will do projectile motion right now.

The athlete will fly in the sky to make some fantastic action like twisting or rotation. The whole time is depending on the height he achieves. And for athlete himself, he must embrace like a ball to keep balance. After making action he will turn down to be ready for getting touch with the pipe again.

So after learning well about these, we find that our model will be divided into two parts, the first is about getting up along with the pipe and the second is about the projectile motion.

\section{Model development}

\subsection{Symbols}

\begin{tabular}{|c|l|}
\hline Symbols & \\
\hline$F_{c}$ & Centripetal Force \\
\hline$f$ & Friction \\
\hline$N$ & Normal Force \\
\hline$\theta$ & The angle corresponding to height \\
\hline$\beta$ & The angle corresponding to the direction of Projectile \\
\hline $\mathrm{R}$ & Radius \\
\hline$v(\theta)$ & The velocity corresponding to height \\
\hline$v_{0}$ & Initial velocity \\
\hline$v_{1}$ & $\begin{array}{l}\text { The additional velocity came from the push on the back } \\
\text { of the snowboard }\end{array}$ \\
\hline$\mu$ & Coefficient of friction \\
\hline$G$ & Gravitational acceleration \\
\hline
\end{tabular}




\begin{tabular}{|c|l|}
\hline$M$ & The mass of the snowboard and the athlete \\
\hline$E_{\max }$ & $\begin{array}{l}\text { The maximum of energy given by the athlete when he } \\
\text { jumps up }\end{array}$ \\
\hline$J_{\min }$ & The minimum of Moment of Inertia \\
\hline$T$ & The time interval of the athlete jumping in the sky \\
\hline$H$ & The highest height gained by the athlete \\
\hline$\sigma$ & The whole angle the athlete can use \\
\hline
\end{tabular}

\subsection{Hypothesis}

1. In the whole process, the model does not consider the existence of air resistance and the wind power.

2. When the athlete jumps up, the model does not consider the complete process about how he step the back of the snowboard.

3. We assume that every athlete have the same initial velocity $v_{0}$, jumped additional velocity $v_{1}$. And it is obvious that $E_{\max }$ and $J_{\min }$ is different for everyone but we assume it is a constant number in the model.

4. We assume the half-pipe is a perfect semicircle instead of an oval, especially for the transition path when the athlete moves up.

\subsection{The deduction of the model}

To build a model of the solution, at first, we apply the Newton second law to express circular motion of the athlete in the half-pipe:

$$
F_{c}=\mathrm{N}-m g \cos \theta=\frac{m v^{2}}{R}
$$

Then, we list the expression of the friction force as follows:

$$
f=\mathrm{N} \mu
$$

Applying the theorem of kinetic energy, we obtain the expression of the decrease in kinetic energy when the athlete is moving up from the bottom of the half-pipe:

$$
\frac{1}{2} m v^{2}-\frac{1}{2} m v_{0}^{2}=\int_{0}^{\theta} f R d \theta+(\mathrm{R}-\mathrm{R} \cos \theta) \mathrm{mg}
$$

Regarding $\theta$ as the independent variable, we differentiate the both sides of equation (3), and then we obtain the equation (4) as follows: 


$$
m v v^{\prime}=\mathrm{f}(\theta) \mathrm{R}+\mathrm{mgR} \sin \theta
$$

Plugging equations (1) and (2) into the equation (4), we obtain:

$$
\nu v^{\prime}=g \mu \mathrm{R} \cos \theta+\mu v^{2}+g \mathrm{R} \sin \theta
$$

Obviously, the format of equation (5) is accord with the format of Bernoulli equation, so we set $s=v^{2}$, and then equation (5) will become:

$$
\frac{d s}{d \theta}=2 v v^{\prime}=2(\mathrm{~g} \mu \mathrm{R} \cos \theta+\mu \mathrm{s}+\mathrm{gR} \sin \theta)
$$

Throwing away the constant term of equation (5), we obtain:

$$
\frac{d s}{d \theta}=2 \mu s
$$

Then we integrate the equation (7) and regard the constant term $\mathrm{C}$ as a function with the independent variable $\theta$, we obtain the particular solution of equation (4) by using the Separation of variables formula:

$$
v^{2}=s=\frac{-2 g R}{1+4 \mu^{2}}\left(\mu \sin \theta+\left(2 \mu^{2}+1\right)\left(\cos \theta-\mathrm{e}^{2 \mu \theta}\right)\right)+\mathrm{ce}^{2 \mu \theta}
$$

Plugging the velocity at the bottom of the half-pipe into the equation (8), we can obtain the velocity equation:

$$
v^{2}=s=\frac{-2 g R}{1+4 \mu^{2}}\left(\mu \sin \theta+\left(2 \mu^{2}+1\right)\left(\cos \theta-\mathrm{e}^{2 \mu \theta}\right)\right)+\mathrm{v}_{0}^{2} \mathrm{e}^{2 \mu \theta}
$$

Now we assume that an athlete will acquire perpendicular velocity $v_{1}$ when he step on the snowboard, and the tangent velocity will be damnified due to the instantaneous friction force, so we suppose the loss rate of the speed is $\lambda$, then the initial velocity of the projectile motion is:

$$
v_{2}=\sqrt{v_{1}^{2}+(\lambda v)^{2}}
$$

So we can obtain the greatest height of the athlete in the air:

$$
h=\frac{\left(\mathrm{v}_{1}^{2}+(\lambda \mathrm{v})^{2}\right) \sin ^{2}\left(\pi-\theta-\arctan \left(\frac{v_{1}}{\lambda v}\right)\right)}{2 g}-R \cos \theta
$$

And the time during "flying" in the air is:

$$
T=\frac{2 \sqrt{v_{1}^{2}+(\lambda v)^{2}} \sin \left(\pi-\theta-\arctan \left(\frac{v_{1}}{\lambda v}\right)\right)}{g}
$$

According to the requirement of the skill, knees should be close to the pit of the stomach, so we can regard the athlete as a rigid body. Then we set the maximum kinetic energy of the revolving rigid body as $E_{\max }$, besides, as the 
athlete should shrink the arm to reduce the moment of inertia, angular velocity can be obtained by best to complete the action, we can set the minimum moment of inertia as $J_{\min }$. Then we obtain the angular velocity:

$$
\omega=\sqrt{\frac{E_{\text {max }}}{J_{\text {min }}}}
$$

So we obtain the angle of the revolving is:

$$
\sigma=\omega T
$$

In conclusion, we obtain the expression of the height $h(\mathrm{R}, \theta)$ and the expression of the angle $\sigma(\mathrm{R}, \theta)$.

\subsection{The solution of the model}

On the basis of the model we obtained above, we analyzed the solution of the model we established, and decided to solve it by using Matlab.

\subsubsection{The function of $\mathrm{T}$}

At first, by consulting the literature, we found that the maximum friction factor of the snowboard course is 0.2 , then we assume that the initial velocity (the velocity of the athlete while he is at the bottom of the half-pipe) is $10 \mathrm{~m} / \mathrm{s}$, the loss rate of the tangential speed is 0.5 .Under these circumstance, we substituted them into the function of height, then we plotted the image of the height by Matlab (Figures 1 and 2) as follows:

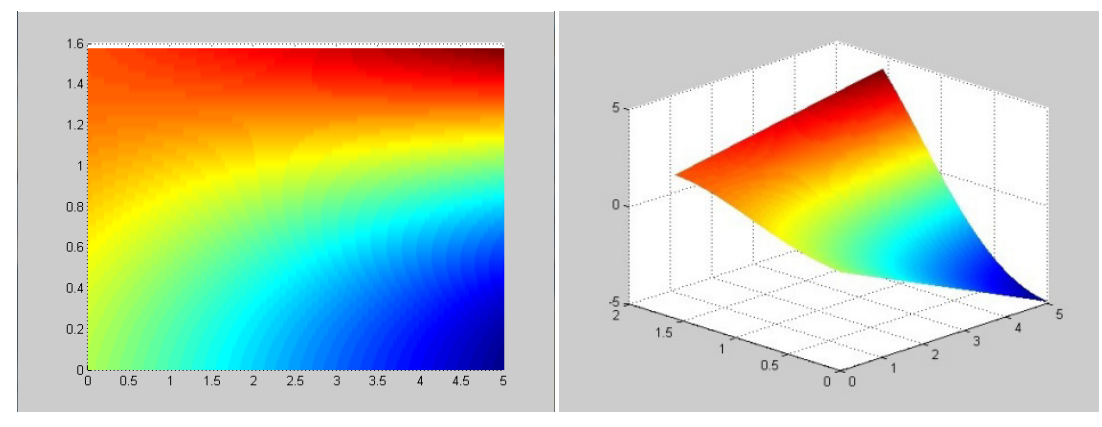

Figure 1: Looking downwards. $\quad$ Figure 2: Maximum height.

These two figures show the same image by different visual angles. The sequence of the height is presented from blue to red as the sequence of the maximum to minimum. We can find out the conclusion obviously by analyzing it. Apparently, in a certain extent of interval, the greatest high emerges in the top right corner (figure 2).

Then, we use the same condition to plot the image of the time. The image is presented as follows (figures 3 and 4): 


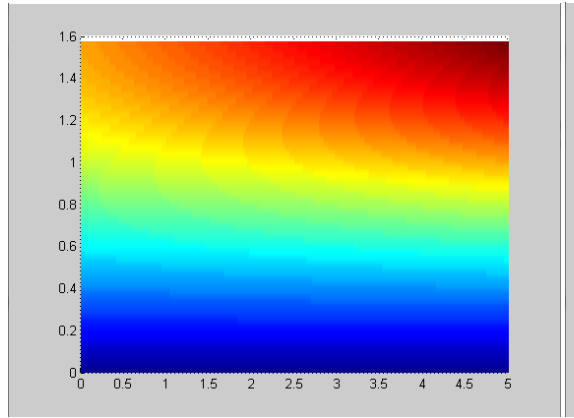

Figure 3: Looking downwards.

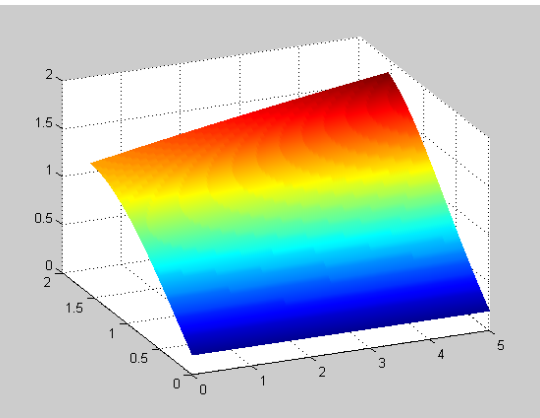

Figure 4: Maximum time.

The two figures show the same image by different visual angles. Obviously, the sequence of the length of time is presented from red to blue as the sequence of the maximum to minimum. Obviously, we can draw a conclusion from these figures that the longest time emerges in the top right corner.

Now we can draw a conclusion, the best opportunity to lift-off of an athlete is the moment when he is at the top of the arch. As for designing the ideal halfpipe, designer should increase the radius of the half-pipe to guarantee the athlete to move up to the top edge of the arc.

To obtain more data of our analysis, we modify the friction factor from 0.2 to 0.05 , and then we plot the superposition image of height by Matlab as follows (figures 5 and 6):

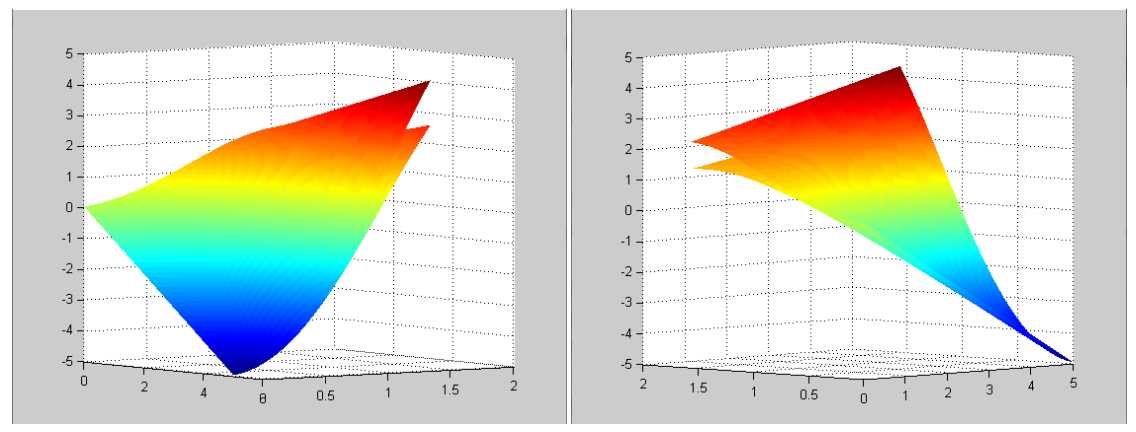

Figure 5: Different friction height. Figure 6: Different friction height.

From the two figures above, we found that the height of the athlete in the air would increase by the reduction of the friction force, which is corresponding with the reality. However, it's obvious that the scope of the increasing height would only be $1 \mathrm{~m}$ approximately.

We also use the Matlab to describe the superposition of time with the same condition (the friction factor is 0.05 ): 


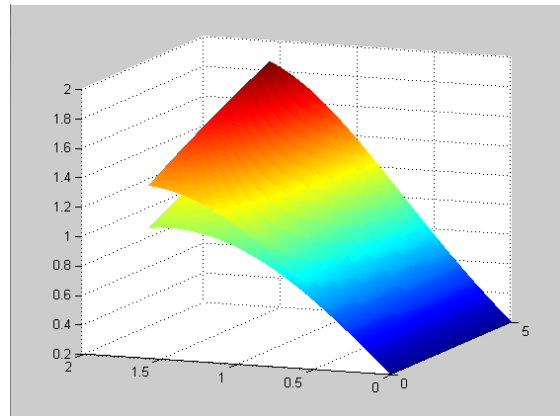

Figure 7: Different friction time.

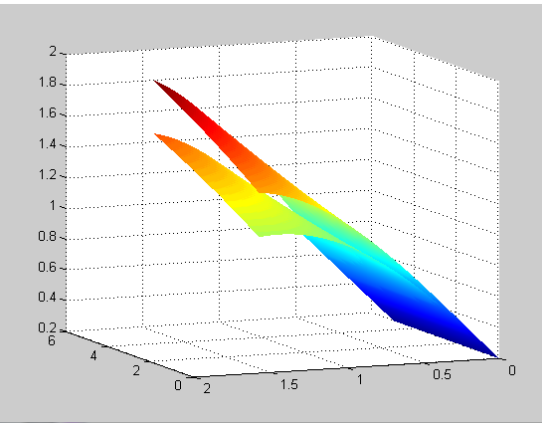

Figure 8: Different friction time.

According to the two figures above, we found that the time of "flying" in the air would increase with the decreasing of the friction factor, which corresponds with the reality. What's more, the time of the athlete "flying" in the air would only increase $0.4 \mathrm{~s}$ approximately.

We tried to change the initial velocity from $10 \mathrm{~m} / \mathrm{s}$ to $15 \mathrm{~m} / \mathrm{s}$, and substituted it into the function. The figures below are the equation described by Matlab (figures 9 and 10):

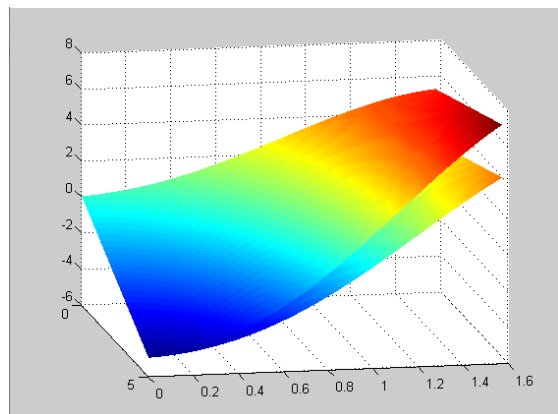

Figure 9: $\quad$ Initial velocity height.

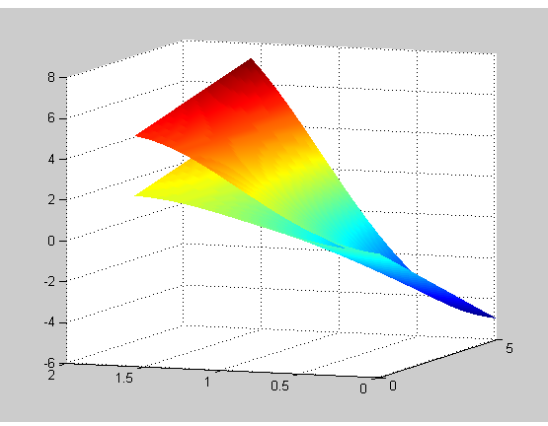

Figure 10: Initial velocity height.

Due to the velocity of $15 \mathrm{~m} / \mathrm{s}$ is the greatest controllable velocity of the athletes, we can know that the greatest height of an athlete can only reach to $7 \mathrm{~m}$, and it will emerge the drop of 3 meters.

Then we plot the image of time by Matlab with the same condition (initial velocity is $15 \mathrm{~m} / \mathrm{s}$ ): 


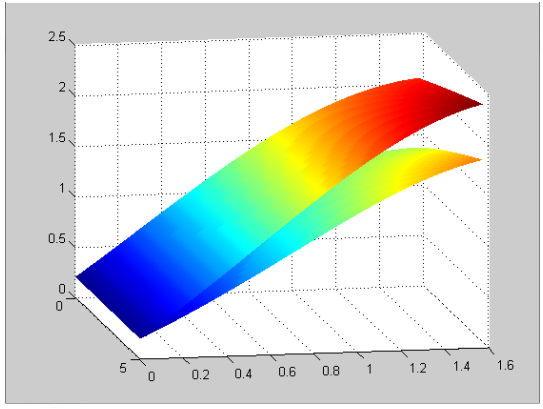

Figure 11: Different initial velocity time.

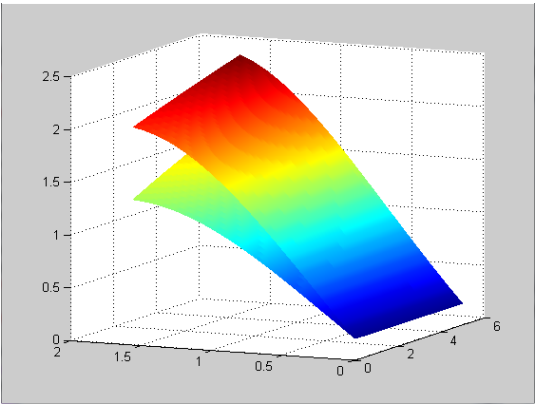

Figure 12: Different initial velocity time.

From the two figures we obtain the information of the change of time. Because $15 \mathrm{~m} / \mathrm{s}$ is the greatest controllable velocity of the athletes, so the greatest time of athlete "flying" in the air will only reach to $2.5 \mathrm{~s}$ approximately.

We also tried to modify the loss rate of tangential velocity to gain more details. Firstly, we change the loss rate of tangential velocity from 0.5 to 1 . We plotted the image to make a comparison between the loss rate of tangential velocity 0.5 and the condition without any loss:
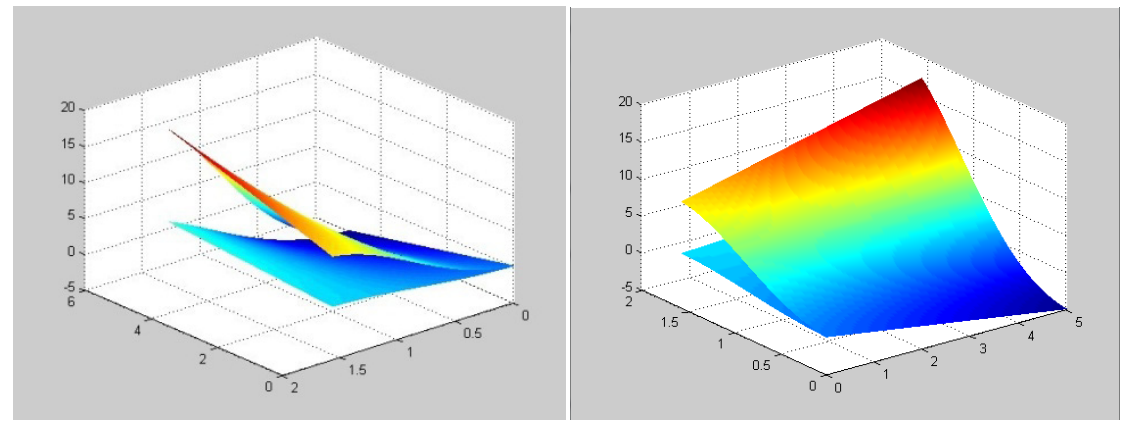

Figure 13: Different loss rate height. Figure 14: Different loss rate height.

The two images display that the height of "flying" in the air would increase tempestuously without the loss of tangential velocity (from $5 \mathrm{~m}$ to $15 \mathrm{~m}$ ), which is contrary to the fact. So it confirms the necessity of setting the loss rate of tangential velocity, which makes the model more close to the reality.

Then we plotted the time images of the same condition (the loss rate of tangential velocity is 1):

The two images displays that the time of "flying" in the air would increase tempestuously without the loss of tangential velocity (from $1.5 \mathrm{~s}$ to $3 \mathrm{~s}$ ), which is also contrary to the fact. It goes a step further to confirm the necessity of setting the loss rate of tangential velocity, which makes the model more close to the reality. 


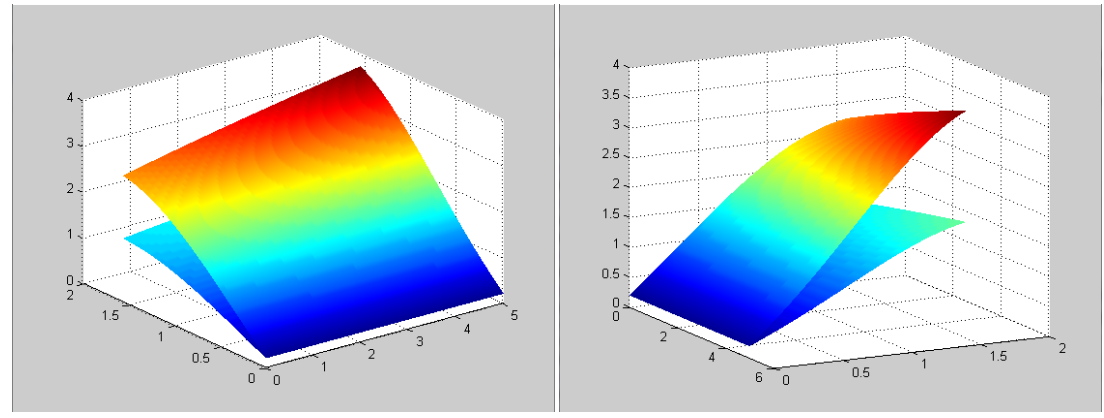

Figure 15: Different loss rate time. Figure 16: Different loss rate time.

\section{Model development}

In this model, we make an assumption in which said the athlete have the same energy when he join up. But actually, different people have different energy. Maybe their energy distribution will be like the normal distribution. We can do more statistics analysis in this field.

The speed of wind is ignored in this model. However, we can add something to consider such effect.

We can learn more about the action detail in jumping. Some research reported that most athletes can't jump right on the side of the snowboard. We can learn more to help them do the perfect job.

\section{References}

[1] Bowden, F.P, Friction on snow and ice. Proceedings of the Royal Society of London, 1953, 217: 462-478.

[2] Colbeck, S. C. The Kinetic Friction of Snow. Journal of Glaciology, 1988, 34(116): 78-86. 